\title{
Refinements on Gap Functions and Optimality Conditions for Vector Quasi-Equilibrium Problems via Image Space Analysis
}

\author{
Jun $\mathrm{Li}^{1}$, Giandomenico Mastroeni ${ }^{2}$
}

\begin{abstract}
By means of some new results on generalized systems, vector quasi-equilibrium problems with a variable ordering relation are investigated from the image perspective. Lagrangian type optimality conditions and gap functions are obtained under mild generalized convexity assumptions on the given problem. Applications to the analysis of error bounds for the solution set of a vector quasi-equilibrium problem are also provided. These results are refinements of several authors' works in recent years and also extend some corresponding results in the literature.
\end{abstract}

Keywords: Image space analysis, Generalized convexity, Generalized systems, Quasi-equilibrium problems

AMS subject classification:90C29, 90C46

\section{Introduction}

A vector quasi-equilibrium problem (for short, VQEP) is a very general model, which includes, as particular cases, (quasi vector) optimization problems (see, for example, [1, 2]), (quasi vector) variational inequalities [3] and (classical) equilibrium problems [4]. Existence results, optimality conditions and gap functions for VQEP have been investigated by many authors (see, for example, $[3,5-7]$, as regards existence results, [8,9], concerning optimality conditions, $[10,11]$, as regards gap functions). In particular, gap functions have shown to be a powerful tool in order to develop algorithms for scalar equilibrium problems in finite-dimensional spaces [12] and find important applications in the analysis of error bounds for VQEP [9].

The purpose of this paper is to investigate a vector quasi-equilibrium problem with a variable ordering relation by using the image space analysis, a general approach, which provides a unifying treatment of several topics concerning optimization problems, from optimality and regularity conditions to sensitivity analysis and penalty methods [13]. By means of some new

\footnotetext{
${ }^{1}$ J. Li, China West Normal University, School of Mathematics and Information, Nanchong, Sichuan 637009, China, e-mail: junli@cwnu.edu.cn

${ }^{2}$ University of Pisa, Department of Computer Science, Largo B. Pontecorvo 3, 56127 Pisa, Italy, e-mail: gmastroeni@di.unipi.it
} 
results on generalized systems [14], we obtain Lagrangian type optimality conditions for VQEP under mild generalized convexity assumptions on the given problem. We also provide gap functions for VQEP and error bounds for the solution set of VQEP under suitable assumptions. These results are refinements of several authors' works in recent years and also extend some corresponding results in the literature (see, for example, $[8,9]$ ) .

The paper is organized as follows. Section 2 is devoted to preliminary results and in particular it is shown that VQEP can be formulated by the impossibility of a suitable generalized system. In Section 3, we present a refinement of the saddle point and KKT-type optimality conditions for VQEP. In Section 4, we deepen the analysis of gap functions associated with VQEP and we provide an application to the error bounds for the solution set under suitable strong monotonicity assumptions.

\section{Preliminary Results}

Let us recall the main definitions and notations, that will be used in the paper. Let $\mathbb{R}^{\ell}$ be the $\ell$ dimensional Euclidean space, where $\ell$ is a given positive integer and set $\mathbb{R}_{+}^{\ell}:=\left\{x:=\left(x_{1}, \cdots, x_{\ell}\right): x_{i} \geq 0, i=1, \cdots, \ell\right\}$. Denote by $\langle\cdot, \cdot\rangle$ and $\|\cdot\|$ the inner product and norm in $\mathbb{R}^{\ell}$.

Let $W$ be a Hausdorff locally convex topological vector space. Denote by $W^{*}$ the topological dual of $W$. A nonempty subset $P \subseteq W$ is said to be a cone if $t P \subseteq P$ for all $t>0$; moreover, if $\mathbf{0} \in P$, then we say that $P$ has apex at the origin. A convex cone $P \subseteq W$, with apex at the origin, is said to be pointed if $P \cap(-P)=\{\mathbf{0}\}$. We denote by $P^{*}:=\left\{x^{*} \in W^{*}:\left\langle x^{*}, x\right\rangle \geq\right.$ $0, \forall x \in P\}$ the positive polar cone of $P \subseteq W$.

Let $M \subset W$ be a nonempty subset. The convex hull, the closure, the interior, the relative interior and the relative boundary of $M$ are denoted by conv $M, \operatorname{cl} M$, int $M$ and ri $M$, and $\operatorname{rbd} M$, respectively. Note that ri $M$ is the interior of $M$ relative to the closed affine hull of $M$ denoted by aff $M$. The set cone $M:=\bigcup_{t>0} t M$ is the cone generated by $M$, with apex at the origin. Let $M \subseteq \mathbb{R}^{m}$. Then, lin $M$ denotes the smallest subspace containing $M$ and $M^{\perp}$ is the orthogonal complement of $M$ given by $M^{\perp}:=\left\{\lambda \in \mathbb{R}^{m}\right.$ : $\langle\lambda, x\rangle=0$ for every $x \in M\}$.

Let $U$ and $Y$ be Hausdorff locally convex topological vector spaces. By $L(U, Y)$, we denote the set of all linear continuous functions from $U$ into $Y$. By $N_{M}(x):=\left\{x^{*} \in W^{*}:\left\langle x^{*}, y-x\right\rangle \leq 0, \forall y \in M\right\}$, we denote the normal cone to $M$ at $x \in M$. For $l \in L(U, Y)$, the value of $l$ at $x$ is denoted by $\langle l, x\rangle$. We recall that, for $\Lambda \in L(U, Y)$, the adjoint operator $\Lambda^{*}: Y^{*} \rightarrow U^{*}$ 
is defined by the equality

$$
\left\langle\Lambda^{*} y^{*}, x\right\rangle=\left\langle y^{*}, \Lambda x\right\rangle, \quad \text { for all } y^{*} \in Y^{*} \text { and } x \in U \text {. }
$$

Definition 2.1 Let $U$ and $Y$ be Banach spaces and $X$ an open subset of $U$. A vector valued function $f: X \rightarrow Y$ is said to be Fréchet differentiable at $\bar{x} \in X$, if there exists a linear continuous operator $\Phi(\bar{x}) \in L(U, Y)$, such that:

$$
\lim _{x \rightarrow \bar{x}} \frac{\|f(x)-f(\bar{x})-\langle\Phi(\bar{x}), x-\bar{x}\rangle\|}{\|x-\bar{x}\|}=0,
$$

where $\|\cdot\|$ is the norm in $U$ and $Y . \Phi(\bar{x})$ is called the Fréchet differential of $f$ at $\bar{x}$. If $f$ is Fréchet differentiable at every $x \in X$, then $f$ is said to be Fréchet differentiable on $X$.

The following definition is due to Borwein and Lewis.

Definition 2.2 ([15]) Let $M$ be a nonempty and convex subset of a Hausdorff locally convex topological vector space $W$.

(1) We say that $x \in M$ is a quasi interior point of $M$, denoted by $x \in q i M$, if cl cone $(M-x)=W$, or equivalently, $N_{M}(x)=\{\mathbf{0}\}$;

(2) We say that $x \in M$ is a quasi relative interior point of $M$, denoted by $x \in \operatorname{qri} M$,

if cl cone $(M-x)$ is a subspace of $W$, or equivalently, $N_{M}(x)$ is a subspace of $W^{*}$.

For any convex set $M$, we have that qi $M \subseteq$ qri $M$, and int $M \neq \emptyset$ implies int $M=$ qri $M[15]$ and int $M=$ qi $M$ [16]. Moreover, qri $\{x\}=\{x\}$, $\forall x \in W$. Similarly, if qi $M \neq \emptyset$, then qi $M=$ qri $M[16,17]$. Moreover, if $W$ is a finite dimensional space, then qi $M=\operatorname{int} M[16]$ and qri $M=\operatorname{ri} M$ [15].

Given $U, Y, Z$ Hausdorff locally convex topological vector spaces, let $X \subseteq$ $U$ be a nonempty subset and $K: X \rightrightarrows X$ be a set-valued mapping. Let $\{C(x): x \in X\}$ be a family of closed, pointed and convex cones in $Y$ with qri $C(x) \neq \emptyset$ for each $x \in X$, and $\{D(x): x \in X\}$ a family of closed and convex cones in $Z$. For each $x \in X$, we will denote $C(x) \backslash\{\mathbf{0}\}$ by $C_{0}(x)$. Let $f: X \times X \rightarrow Y$ with $f(x, x)=\mathbf{0}$ for each $x \in X$. We consider the following vector quasi-equilibrium problem (for short, VQEP) with a variable ordering relation: find $x \in K(x)$ such that

$$
f(x, y) \nsupseteq C_{0}(x) \mathbf{0}, \forall y \in K(x),
$$


where the relations $\geq$ and $¥$ are defined by the following:

$$
x \geq_{P} y \quad \Leftrightarrow \quad x-y \in P, \quad \forall x, y \in Y
$$

and

$$
x \nsupseteq_{P} y \quad \Leftrightarrow \quad x-y \notin P, \quad \forall x, y \in Y,
$$

where $P \subseteq Y$ is a convex cone. If we replace $C_{0}(x)$ by qri $C(x)(\neq \emptyset)$, qi $C(x)(\neq \emptyset)$, ri $C(x)(\neq \emptyset)$ and int $C(x)(\neq \emptyset)$ in $(1)$, respectively, then we obtain the definitions of quasi relatively weak VQEP (for short, qr-weak VQEP), quasi weak VQEP (for short, q-weak VQEP), relatively weak VQEP (for short, r-weak VQEP) and weak VQEP.

The introduction of q-weak VQEP allows us to consider convex cones $C(x)$ with empty topological interior in the infinite dimensional case. A classic example is given by

$$
C(x)=L_{+}^{p}:=\left\{u \in L^{p}(\Gamma): u(t) \geq 0 \quad \text { for a.e. } t \in \Gamma\right\},
$$

where $(1<p<+\infty)$ and $\Gamma$ is an open and bounded set in $\mathbb{R}^{n}$. In this case

$$
\text { qi } C(x)=\left\{u \in L^{p}(\Gamma): u(t)>0 \quad \text { for a.e. } t \in \Gamma\right\} .
$$

Similarly qr-weak VQEP allows us to consider convex cones $C(x)$ with empty quasi interior both in the infinite and finite dimensional case. We recall that both qiC $(x)$ and qriC $(x)$ are convex cones provided that $C(x)$ is a convex cone. For an extensive discussion on the use of ordering cones with empty interiors in vector optimization, we refer to [18] and references therein.

We need the following lemmas. Statements (i) and (ii) of the following lemma can be found in Theorem 3.10 of [15], Theorem 2.4 and Proposition 2.6 of [19], Theorem 5.3 and Proposition 5.3 of [14], respectively.

Lemma 2.1 Let $M$ be a closed and convex cone of a Hausdorff locally convex topological vector space $W$ with $\operatorname{cl}(M-M)=W$. Then

(i) $x \in \operatorname{qri} M \quad \Leftrightarrow \quad\left\langle\lambda^{*}, x\right\rangle>0, \forall \lambda^{*} \in M^{*} \backslash\{\mathbf{0}\}$;

(ii) qri $M=$ qi $M$.

Statements (i)-(v), (vi) and (vii) of the following lemma can be found in $[15-17,20]$ and [9], respectively. 
Lemma 2.2 Let $M$ and $N$ be convex subsets of a Hausdorff locally convex topological vector space $W$. Then the following statements are true:

(i) $\operatorname{qri}(t M)=t$ qri $M, \quad \forall t \in \mathbb{R}$;

(ii) $t$ qri $M+(1-t) M \subseteq$ qri $M, \forall t \in] 0,1]$; hence, qri $M$ is a convex set;

(iii) $\operatorname{cl}$ qri $M=\operatorname{cl} M$;

(iv) $\operatorname{qri}(M \times N)=\operatorname{qri} M \times \operatorname{qri} N$;

(v) $\operatorname{qri}($ qri $M)=\operatorname{qri} M$;

(vi) If $M$ is a convex cone, then $\operatorname{qri} M+M=$ qri $M$;

(vii) If qri $M \neq \emptyset$, then (qri $M)^{*}=M^{*}$.

Lemma 2.3 [20, Theorem 2.12] Let $M$ be a convex subset of a Banach space $W$. Then $\operatorname{ri} M \subseteq \operatorname{qri} M$. Moreover, any of the following conditions guarantees that $\operatorname{ri} M=\operatorname{qri} M$ :

(i) $W$ is finite dimensional;

(ii) $\operatorname{int} M$ is nonempty;

(iii) $\operatorname{ri} M$ is nonempty.

Lemma 2.4 [21, Lemma 4.2.1 and Remark 4.2.2, Chapter III ] Let $B$ be a convex set in $\mathbb{R}^{m}$ and $x \in \operatorname{rbd} B$. Then $B$ admits a supporting hyperplane at $x$ and its normal vector belongs to aff $(B-x)$.

We will consider the following definitions of $P$-function and strict $P$-function of a vector valued function.

Definition 2.3 Let $\mathbf{K}$ be a convex subset of $X$ and $P$ be a convex cone in $Y$, with apex at the origin, and $\mathbf{f}: X \rightarrow Y$.

(1) $\mathbf{f}$ is a P-function on $\mathbf{K}$, if, for any $\left.x_{1}, x_{2} \in \mathbf{K}, \alpha \in\right] 0,1[$,

$$
\alpha \mathbf{f}\left(x_{1}\right)+(1-\alpha) \mathbf{f}\left(x_{2}\right)-\mathbf{f}\left(\alpha x_{1}+(1-\alpha) x_{2}\right) \in P
$$

(2) $\mathbf{f}$ is a strict P-function on $\mathbf{K}$, iff, for any $\left.x_{1} \neq x_{2} \in \mathbf{K}, \alpha \in\right] 0,1[$,

$$
\alpha \mathbf{f}\left(x_{1}\right)+(1-\alpha) \mathbf{f}\left(x_{2}\right)-\mathbf{f}\left(\alpha x_{1}+(1-\alpha) x_{2}\right) \in \operatorname{qri} P,
$$

provided that qri $P \neq \emptyset$. 
Clearly, $\mathbf{f}$ is a (res., strict) $P$-function on $\mathbf{K}$, iff $-\mathbf{f}$ is a (res., strict) $P$-function on $\mathbf{K}$. When $Y:=\mathbb{R}$ and $P:=\mathbb{R}_{+}$, the previous definition collapses to that of the classical convexity.

Next result shows a characterization of a $P$-function in terms of the properties of its $P$-epigraph.

Proposition 2.1 [2, Proposition 6.2] Let $P$ be a convex cone in $Y$, with apex at the origin. Then, $\mathbf{f}: X \rightarrow Y$ is a P-function on the convex set $\mathbf{K} \subseteq X$, iff

$$
\operatorname{epi}_{P} \mathbf{f}_{\mid \mathbf{K}}:=\{(x, y) \in X \times Y: y \in \mathbf{f}(x)+P, x \in \mathbf{K}\}
$$

is a convex set. Moreover, if $P$ is a closed and convex cone, then $\mathbf{f}$ is a $P$-function on $\mathbf{K}$, iff $\theta \circ \mathbf{f}$ is a convex function on $\mathbf{K}$, for every $\theta \in P^{*}$.

It is easy to see that, if $\mathbf{f}$ is a strict $P$-function on $\mathbf{K}$, then $\mathbf{f}(\mathbf{K})+$ qri $P$ is convex by Lemma 2.2 (ii). The concept of convexity has been generalized in several ways. In particular, we recall the following definition [22].

Definition 2.4 Given a convex set $\mathcal{A} \subseteq Y$, a function $\phi: X \rightarrow Y$ is said to be generalized $\mathcal{A}$-subconvexlike on $X$ if cone $(\phi(X))+$ qri $\mathcal{A}$ is convex.

Throughout this paper, we suppose that the feasible set of problem (1) is defined by

$$
K(x):=\left\{y \in X: g(x, y) \geq_{D(x)} \mathbf{0}\right\}
$$

where $g: X \times X \rightarrow Z$.

We preliminarily observe that VQEP can be cast under the form of the impossibility of a generalized system [13, 14, 23, 24] defined by

$$
F(x ; y) \in \mathcal{H}(x), \quad y \in X, \quad x \in \mathcal{Y},
$$

where $V$ is a Hausdorff topological vector space, $\mathcal{H}(x) \subseteq V$, for every $x \in \mathcal{Y}$, $X$ is a subset of $U, \mathcal{Y}$ is a parameter set and $F: U \times \mathcal{Y} \rightarrow V$ is a mapping. Indeed, note that, $x \in K(x)$ solves VQEP, iff the following system (in the unknown $y$ ) is impossible:

$$
f(x, y) \geq_{C_{0}(x)} \mathbf{0}, \quad g(x, y) \geq_{D(x)} \mathbf{0}, \quad y \in X
$$

Let $V:=Y \times Z$; define the mapping $F$ and the set $\mathcal{H}(x)$ by

$$
F(x ; y):=(f(x, y), g(x, y)), \quad \mathcal{H}(x):=C_{0}(x) \times D(x),
$$


and set $\mathcal{Y}:=\{x \in X: x \in K(x)\}$. It is easily seen that (3) is equivalent to (2).

Similarly, if we set

(i) $\mathcal{H}(x):=\operatorname{qri} C(x) \times D(x)$,

(ii) $\mathcal{H}(x):=$ qi $C(x) \times D(x)$,

(iii) $\mathcal{H}(x):=\operatorname{ri} C(x) \times D(x)$,

(iv) $\mathcal{H}(x):=\operatorname{int} C(x) \times D(x)$,

then (2) is impossible iff $x \in K(x)$ solves qr-weak VQEP, q-weak VQEP, r-weak VQEP and weak VQEP, respectively.

We recall that the generalized system (2) is said to be image convex, if for all $x \in \mathcal{Y}$ such that (2) is impossible, the sets $F(x ; X)$ and $\mathcal{H}(x)$ are linearly separable. Let us recall some of the main results obtained in [14] and that will be used throughout the paper.

Theorem 2.1 [14, Theorem 4.2] Let $x \in X$ and let $\mathcal{A}(x)$ be a convex cone in $V$, with apex at the origin, such that

$$
\mathcal{H}(x)-\mathcal{A}(x)=\mathcal{H}(x) .
$$

Assume that $\mathcal{H}(x)$ is a convex cone and that $F(x ; \cdot)$ is generalized $\mathcal{A}(x)$ subconvexlike on $X$. If $V$ is finite dimensional with $0 \notin \mathrm{ri} \mathcal{H}(x)$, or if int $\mathcal{A}(x) \neq \emptyset$, then system (2) is image convex.

Theorem 2.2 [14, Theorem 4.3] Let $x \in X$ and $\mathcal{A}(x)$ be a convex cone in $V$, with apex at the origin, such that qri $\mathcal{A}(x) \neq \emptyset$ and $\operatorname{cl} \mathcal{A}(x)=-\operatorname{cl} \mathcal{H}(x)$. If, additionally, $F(x ; \cdot)$ is generalized $\mathcal{A}(x)$-subconvexlike on $X$, and $0 \notin$ qi $[\mathrm{cl}(\operatorname{cone}(F(x, X)+\mathcal{A}(y)))]$, then system $(2)$ is image convex.

\section{Lagrangian Type Optimality Conditions}

Let $x \in K(x)$ be fixed and consider the generalized Lagrangian function associated with VQEP defined by $\mathcal{L}_{x}: X \times(C(x))^{*} \times(D(x))^{*} \rightarrow \mathbb{R}$,

$\mathcal{L}_{x}(y, \theta, \lambda):=-[\langle\theta, f(x, y)\rangle+\langle\lambda, g(x, y)\rangle], \forall(y, \theta, \lambda) \in X \times(C(x))^{*} \times(D(x))^{*}$.

Definition 3.1 The point $\left(x, \theta^{*}, \lambda^{*}\right) \in X \times(C(x))^{*} \times(D(x))^{*}$ is said to be a saddle point of the generalized Lagrangian function $\mathcal{L}_{x}$ on $X \times(C(x))^{*} \times$ $(D(x))^{*}$ if the following inequalities hold:

$\mathcal{L}_{x}(x, \theta, \lambda) \leq \mathcal{L}_{x}\left(x, \theta^{*}, \lambda^{*}\right) \leq \mathcal{L}_{x}\left(y, \theta^{*}, \lambda^{*}\right), \quad \forall(y, \theta, \lambda) \in X \times(C(x))^{*} \times(D(x))^{*}$. 
Theorem 3.1 Suppose that $x \in K(x)$ is a solution to VQEP (res., qr-weak VQEP, q-weak VQEP, weak VQEP, and r-weak VQEP with $Y$ being a Banach space) and that (2) is image convex. Then, there exist $\theta^{*} \in(C(x))^{*}$ and $\lambda^{*} \in(D(x))^{*},\left(\theta^{*}, \lambda^{*}\right) \neq \mathbf{0}$, such that $\left(x, \theta^{*}, \lambda^{*}\right)$ is a saddle point for $\mathcal{L}_{x}$ on $X \times(C(x))^{*} \times(D(x))^{*}$.

Proof Clearly, $\left(C_{0}(x)\right)^{*}=(C(x))^{*}$ and by Lemma $2.2(\operatorname{vii}),(\operatorname{qri} C(x))^{*}=$ $(C(x))^{*}$, provided that qri $\left.C(x)\right)^{*} \neq \emptyset$. If $Y$ is a Banach space, then from Lemmas 2.2 (vii) and 2.3 (iii) it follows that $(\operatorname{ri} C(x))^{*}=(\operatorname{qri} C(x))^{*}=$ $(C(x))^{*}$. Since $(2)$ is image convex, then there exist $\theta^{*} \in(C(x))^{*}$ and $\lambda^{*} \in(D(x))^{*},\left(\theta^{*}, \lambda^{*}\right) \neq \mathbf{0}$, such that

$$
\left\langle\theta^{*}, f(x, y)\right\rangle+\left\langle\lambda^{*}, g(x, y)\right\rangle \leq 0, \quad \forall y \in X .
$$

Since $x \in K(x)$, one has $\left\langle\lambda^{*}, g(x, x)\right\rangle \geq \mathbf{0}$, so that (7) implies $\left\langle\lambda^{*}, g(x, x)\right\rangle=$ 0 . From the assumption $f(x, x)=\mathbf{0}$ it follows that

$$
0=\mathcal{L}_{x}\left(x, \theta^{*}, \lambda^{*}\right) \leq \mathcal{L}_{x}\left(y, \theta^{*}, \lambda^{*}\right), \forall y \in X
$$

The inequality

$$
\mathcal{L}_{x}(x, \theta, \lambda) \leq \mathcal{L}_{x}\left(x, \theta^{*}, \lambda^{*}\right), \quad \forall(\theta, \lambda) \in(C(x))^{*} \times(D(x))^{*},
$$

i.e.,

$$
-\langle\lambda, g(x, x)\rangle \leq-\left\langle\lambda^{*}, g(x, x)\right\rangle, \forall \lambda \in(D(x))^{*},
$$

is equivalent to

$$
\langle\lambda, g(x, x)\rangle \geq 0, \forall \lambda \in(D(x))^{*},
$$

which is fulfilled since $g(x, x) \in D(x)$.

Remark 3.1 Theorem 3.1 generalizes to qr-weak VQEP Theorem 4.1 in [9].

Theorems 2.1 and 2.2 lead us to obtain the following result.

Theorem 3.2 Let $V:=Y \times Z$ and $x \in K(x)$ be a solution to VQEP (res., qr-weak VQEP, q-weak VQEP) and let $F(x ; y):=(f(x, y), g(x, y))$.

Assume that $F(x ; \cdot)$ is generalized $(C(x) \times D(x))$-subconvexlike on $X$ and any of the following conditions holds:

(i) $V$ is finite dimensional,

(ii) $\operatorname{int}(C(x) \times D(x)) \neq \emptyset$, 
(iii) $\mathbf{0} \notin$ qi $[\operatorname{cl}(\operatorname{cone}(F(x, X)-(C(x) \times D(x))))]$ and qri $D(x) \neq \emptyset$.

Then, there exist $\theta^{*} \in(C(x))^{*}$ and $\lambda^{*} \in(D(x))^{*},\left(\theta^{*}, \lambda^{*}\right) \neq \mathbf{0}$, such that $\left(x, \theta^{*}, \lambda^{*}\right)$ is a saddle point for $\mathcal{L}_{x}$ on $X \times(C(x))^{*} \times(D(x))^{*}$.

Proof Set $\mathcal{H}(x):=\mathcal{C}(x) \times D(x)$, where $\mathcal{C}(x):=C_{0}(x)$, qri $C(x)$, qi $C(x)$, and set

$\mathcal{A}(x):=-(C(x) \times D(x))=-\operatorname{cl} \mathcal{H}(x)$ (by Lemma $2.2($ iii)). From Lemma 2.2 (vi) we can easily check assumption (5) holds and since $C(x)$ is a closed convex pointed cone, it follows that $\mathbf{0} \notin \operatorname{qri} \mathcal{H}(x)=\operatorname{qri} \mathcal{C}(x) \times \operatorname{qri} D(x)$ (by Lemma 2.2 (iv) and (v)). Recalling that if $V$ is finite dimensional, then qri $\mathcal{H}(x)=\operatorname{ri} \mathcal{H}(x) \neq \emptyset$, under assumption (i) or (ii) Theorem 2.1 allows us to prove that system (2) is image convex. If assumption (iii) holds, then Theorem 2.2 directly yields that system (2) is image convex. By Theorem 3.1 we complete the proof.

Now we consider optimality conditions under the generalized Slater condition, i.e.,

$$
\mathbf{0} \in \operatorname{qri}[\operatorname{conv}(g(x, X)-D(x))] .
$$

Regularity conditions for optimization problems, based on the notion of quasi relative interior, have been brought to the attention of the research community in the pioneering works $[17,25]$ and subsequently developed in $[19,26]$.

Under analogous assumptions of the previous theorem, next result ensures that the multiplier associated with the operator $f$ in the saddle point condition is non zero, in case $V$ is finite dimensional. The result extends Theorem 3.6 in [27].

Theorem 3.3 Let $V$ be finite dimensional, $x \in K(x)$ be a solution to qr-weak VQEP (or equivalently, r-weak VQEP) and $F(x ; \cdot)$ be generalized $-(C(x) \times D(x))$-subconvexlike on $X$. Assume, additionally, that (8) holds. Then, there exist $\theta^{*} \in(C(x))^{*} \backslash\{\mathbf{0}\}$ and $\lambda^{*} \in(D(x))^{*}$ such that $\left(x, \theta^{*}, \lambda^{*}\right)$ is a saddle point for $\mathcal{L}_{x}$ on $X \times(C(x))^{*} \times(D(x))^{*}$.

Proof Since $V$ is finite dimensional, qr-weak VQEP collapses to r-weak VQEP. From Lemma 2.2 (iv) we have ri $(C(x) \times D(x))=\operatorname{ri} C(x) \times \operatorname{ri} D(x)$. We note that $x \in K(x)$ solves qr-weak VQEP if and only if

$$
F(x ; X) \cap(\operatorname{ri} C(x) \times D(x))=\emptyset .
$$

We claim that

$$
\mathbf{0} \notin \text { cone } F(x ; X)-\operatorname{ri}(C(x) \times D(x))
$$


Suppose to the contrary that (9) is not true. Then there is $a \in$ cone $F(x ; X)$ with $a:=t b$ such that $a \in \operatorname{ri}(C(x) \times D(x)) \subseteq \operatorname{ri} C(x) \times D(x)$, where $t \geq 0$ and $b \in F(x ; X)$. Since $C(x)$ is a closed, pointed and convex cone, it is impossible for $t=0$. As a consequence, $t>0$ and it follows that $b \in \frac{1}{t}(\operatorname{ri} C(x) \times$ $D(x))=\operatorname{ri}\left(\frac{1}{t} C(x)\right) \times\left(\frac{1}{t} D(x)\right)=\operatorname{ri} C(x) \times D(x)$, where the first equality follows from Lemma $2.2(\mathrm{i})$. This is a contradiction. By assumption, the set cone $F(x ; X)-\operatorname{ri}(C(x) \times D(x))$ is convex and so the following equalities hold:

$$
\begin{aligned}
\text { conv cone } F(x ; X)-\operatorname{ri}(C(x) \times D(x)) & =\operatorname{conv}[\operatorname{cone} F(x ; X)-\operatorname{ri}(C(x) \times D(x))] \\
& =\operatorname{cone} F(x ; X)-\operatorname{ri}(C(x) \times D(x)) .
\end{aligned}
$$

Therefore, from (9) it follows that

$\mathbf{0} \notin \operatorname{ri}($ conv cone $F(x ; X))-\operatorname{ri}(C(x) \times D(x))=\operatorname{ri}($ conv cone $F(x ; X)-(C(x) \times D(x)))$

so that, $\mathbf{0} \in \operatorname{rbd}($ conv cone $F(x ; X)-(C(x) \times D(x)))$, since $\mathbf{0} \in$ conv cone $F(x ; X)-$ $(C(x) \times D(x))$. Applying Lemma 2.4, it follows that there is $(0,0) \neq$ $\left(\theta^{*}, \lambda^{*}\right) \in \operatorname{lin}[\operatorname{conv}$ cone $F(x ; X)-(C(x) \times D(x))]$ such that

$$
\left\langle\theta^{*}, u\right\rangle+\left\langle\lambda^{*}, v\right\rangle \leq 0, \quad \forall(u, v) \in \text { conv cone } F(x ; X)-(C(x) \times D(x)) .
$$

This yields system (2) is image convex and by Theorem $3.1,\left(x, \theta^{*}, \lambda^{*}\right) \in$ $X \times(C(x))^{*} \times(D(x))^{*}$ is a saddle point for $\mathcal{L}_{x}$ on $X \times(C(x))^{*} \times(D(x))^{*}$. We have only to prove that $\theta^{*} \neq \mathbf{0}$. Suppose to the contrary that $\theta^{*}=\mathbf{0}$. Then

$$
\mathbf{0} \neq \lambda^{*} \in \operatorname{lin}[\operatorname{conv} g(x, X)-D(x)]
$$

and from (10) it follows that

$$
\left\langle\lambda^{*}, v\right\rangle \leq 0, \quad \forall v \in \operatorname{conv} g(x, X)-D(x) .
$$

By assumption (8), i.e., $\mathbf{0} \in \mathrm{ri}[\operatorname{conv} g(x, X)-D(x)]$, there exists a neighborhood $U$ of $\mathbf{0} \in Z$ such that

$$
\Gamma:=U \cap \operatorname{lin}[\operatorname{conv} g(x, X)-D(x)] \subseteq[\operatorname{conv} g(x, X)-D(x)] .
$$

By (11), we have that $t \lambda^{*} \in \Gamma$ for $|t|<\epsilon$, where $\epsilon$ is a positive number sufficiently small. Then, (12) yields

$$
t\left\|\lambda^{*}\right\|^{2}=t\left\langle\lambda^{*}, \lambda^{*}\right\rangle \leq 0, \quad \forall t \text { with }|t|<\epsilon,
$$

which is a contradiction since $\lambda^{*} \neq \mathbf{0}$. This completes the proof.

We now give some sufficient optimality conditions for VQEP and qr-weak VQEP. 
Theorem 3.4 The following assertions are true:

(i) Suppose that qri $D(x) \neq \emptyset, \operatorname{cl}(C(x)-C(x))=Y, \operatorname{cl}(D(x)-D(x))=Z$ and that (8) holds.

If, additionally, there exist $\theta^{*} \in(C(x))^{*}$ and $\lambda^{*} \in(D(x))^{*},\left(\theta^{*}, \lambda^{*}\right) \neq$ $\mathbf{0}$, such that $\left(x, \theta^{*}, \lambda^{*}\right)$ is a saddle point for $\mathcal{L}_{x}$ on $X \times(C(x))^{*} \times$ $(D(x))^{*}$, then $x$ is a solution to qr-weak VQEP;

(ii) Suppose that qri $D(x) \neq \emptyset, \operatorname{cl}(C(x)-C(x))=Y$ and that (8) holds.

If, additionally, there exist $\theta^{*} \in(C(x))^{*}$ and $\lambda^{*} \in(D(x))^{*}$ such that $\left(x, \theta^{*}, \lambda^{*}\right)$ is a saddle point for $\mathcal{L}_{x}$ on $X \times(C(x))^{*} \times(D(x))^{*}$ and there exist $\bar{x} \in X$ such that $\mathcal{L}_{x}\left(\bar{x}, \theta^{*}, \lambda^{*}\right)>0$, then $x$ is a solution to qr-weak VQEP;

(iii) If there exist $\theta^{*} \in \mathrm{qi}(C(x))^{*}$ and $\lambda^{*} \in(D(x))^{*}$ such that $\left(x, \theta^{*}, \lambda^{*}\right)$ is a saddle point for $\mathcal{L}_{x}$ on $X \times(C(x))^{*} \times(D(x))^{*}$, then $x$ is a solution to VQEP.

Proof (i) Let $\left(x, \theta^{*}, \lambda^{*}\right)$ be a saddle point for $\mathcal{L}_{x}$ on $X \times(C(x))^{*} \times(D(x))^{*}$, i.e.,

$$
\begin{aligned}
& -[\langle\theta, f(x, x)\rangle+\langle\lambda, g(x, x)\rangle] \leq-\left[\left\langle\theta^{*}, f(x, x)\right\rangle+\left\langle\lambda^{*}, g(x, x)\right\rangle\right] \\
\leq \quad & -\left[\left\langle\theta^{*}, f(x, y)\right\rangle+\left\langle\lambda^{*}, g(x, y)\right\rangle\right], \forall(y, \theta, \lambda) \in X \times(C(x))^{*} \times(D(y))^{*}
\end{aligned}
$$

By the first inequality in (13) we have

$$
-\langle\lambda, g(x, x)\rangle \leq-\left\langle\lambda^{*}, g(x, x)\right\rangle, \quad \forall(\theta, \lambda) \in(C(x))^{*} \times(D(x))^{*} .
$$

Notice that $D(x)=\left((D(x))^{*}\right)^{*}$ (see, [28]), since $D(x)$ is a closed and convex cone in $Z$. Letting $\lambda:=\mathbf{0}$ in (14) leads to $\left\langle\lambda^{*}, g(x, x)\right\rangle \leq 0$. We first prove that $x \in K(x)$, i.e., $g(x, x) \geq_{D(x)} \mathbf{0}$. Ab absurdo, suppose that $g(x, x) \notin D(x)=\left((D(x))^{*}\right)^{*}$. Then there exists $\bar{\lambda} \in(D(x))^{*}$ such that $\langle\bar{\lambda}, g(x, x)\rangle<0$. Since $t \bar{\lambda} \in(D(x))^{*}$ for any $t>0$, then $-t\langle\bar{\lambda}, g(x, x)\rangle \rightarrow$ $+\infty$, which contradicts (14). Since $x \in K(x)$, one has $\left\langle\lambda^{*}, g(x, x)\right\rangle \geq 0$ and so $\left\langle\lambda^{*}, g(x, x)\right\rangle=0$. It follows from the fact $f(x, x)=\mathbf{0}$ and the first inequality in (13) that $\left\langle\theta^{*}, f(x, x)\right\rangle+\left\langle\lambda^{*}, g(x, x)\right\rangle=0$. Consequently, the second inequality in (13) yields (7). By (8), similar to the proof of Proposition 5.4 in [14], we can show that $\theta^{*} \neq \mathbf{0}$. Since $\theta^{*} \neq \mathbf{0}$, from Lemma 2.1 we obtain

$$
\left\langle\theta^{*}, u\right\rangle+\left\langle\lambda^{*}, v\right\rangle>0, \quad \forall(u, v) \in \mathcal{H}(x)=(\operatorname{qri} C(x)) \times D(x) .
$$


It follows that (2) is impossible and $x$ is a solution to qr-weak VQEP.

(ii) As in the proof of (i) we can show that (7) holds and $x \in K(x)$. We now prove that $\theta^{*} \neq \mathbf{0}$. We preliminarily note that, by the assumption $\mathcal{L}_{x}\left(\bar{x}, \theta^{*}, \lambda^{*}\right)>0$, it follows that

$$
\left\langle\theta^{*}, \bar{u}\right\rangle+\left\langle\lambda^{*}, \bar{v}\right\rangle<0,
$$

where $\bar{u}=f(x, \bar{x}), \bar{v}=g(x, \bar{x})$. Suppose to the contrary that $\theta^{*}=\mathbf{0}$ in $(7)$. Then, $\lambda^{*} \neq \mathbf{0}$ and

$$
\left\langle\lambda^{*}, v\right\rangle \leq 0, \quad \forall v \in g(x, X),
$$

which implies that

$$
\left\langle\lambda^{*}, v\right\rangle \leq 0, \quad \forall v \in \operatorname{conv}[g(x, X)-D(x)],
$$

with strict inequality for $v=\bar{v}$. Then,

$$
\lambda^{*} \in N_{M}(\mathbf{0}) \text { and }-\lambda^{*} \notin N_{M}(\mathbf{0}),
$$

where we have set $M:=\operatorname{conv}[g(x, X)-D(x)]$ (note that $\mathbf{0} \in M$, since $x \in K(x)$ ). Therefore $N_{M}(\mathbf{0})$ is not a linear subspace which contradicts (8), recalling the definition of quasi relative interior points.

Thus, we have proved that $\theta^{*} \neq \mathbf{0}$ in (7). The inequality

$$
\left\langle\theta^{*}, f(x, y)\right\rangle \leq\left\langle\theta^{*}, f(x, y)\right\rangle+\left\langle\lambda^{*}, g(x, y)\right\rangle \leq 0, \quad \forall y \in K(x),
$$

yields that $x$ is a solution of qr-weak VQEP. Indeed, suppose to the contrary that $x$ is not a solution of qr-weak VQEP, i.e., there exists $\tilde{y} \in K(x)$ such that, $f(x, \tilde{y}) \in$ qri $C(x)$. Then, by Lemma 2.1 we obtain that

$$
\left\langle\theta^{*}, f(x, \tilde{y})\right\rangle>0,
$$

a contradiction.

(iii) Similar to the proof of (i).

Remark 3.2 Theorem 3.2 provides the existence of a saddle point of the generalized Lagrangian function under one of the weakest generalized convexity assumptions on the functions involved in VQEP and qr-weak VQEP. Theorem 3.4 is closely related to Corollary 4.1 in [9].

Example 3.1 Let $X:=\mathbb{R}_{+}^{2}, Y:=\mathbb{R}^{2}, C(x):=\left(-\mathbb{R}_{+}\right) \times \mathbb{R}_{+}, D(x):=\mathbb{R}_{+}$, $f(x, y):=\left(f_{1}(x, y), f_{2}(x, y)\right)$ and $g(x, y):=y_{1}^{3}-x_{1}^{2}$, where $f_{1}(x, y):=y_{1}-x_{1}$, $f_{2}(x, y):=y_{2}-x_{2}, x:=\left(x_{1}, x_{2}\right)$ and $y:=\left(y_{1}, y_{2}\right)$. Then,

$$
F(x ; y)=(f(x, y), g(x, y))=\left(y_{1}-x_{1}, y_{2}-x_{2}, y_{1}^{3}-x_{1}^{2}\right) .
$$


Let $x:=\left(x_{1}, x_{2}\right):=(0,0) \in K(x)=\left\{\left(y_{1}, y_{2}\right) \in \mathbb{R}_{+}^{2}: y_{1} \geq 0\right\}$. We have

$$
\begin{aligned}
F(x ; X) & =\left\{\left(u_{1}, u_{2}, v\right) \in \mathbb{R}^{3}: u_{1}=y_{1}, u_{2}=y_{2}, v=y_{1}^{3},\left(y_{1}, y_{2}\right) \in \mathbb{R}_{+}^{2}\right\} \\
& =\left\{\left(u_{1}, u_{2}, v\right) \in \mathbb{R}^{3}: u_{1} \geq 0, u_{2} \geq 0, v=u_{1}^{3}\right\}
\end{aligned}
$$

and so

$$
\begin{aligned}
F(x ; X)-(C(x) \times D(x)) & =F(x ; X)+\left(\mathbb{R}_{+} \times\left(-\mathbb{R}_{+}\right) \times\left(-\mathbb{R}_{+}\right)\right) \\
& =\left\{\left(u_{1}, u_{2}, v\right) \in \mathbb{R}^{3}: u_{1} \geq 0, v \leq u_{1}^{3}\right\} .
\end{aligned}
$$

Note that the previous set is nonconvex. Moreover,

$$
\begin{aligned}
\operatorname{cone}(F(x ; X))+\operatorname{qri}[-(C(x) \times D(x))] & =\operatorname{cone}(F(x ; X))+\operatorname{int}\left[\mathbb{R}_{+} \times\left(-\mathbb{R}_{+}\right) \times\left(-\mathbb{R}_{+}\right)\right] \\
& =\left\{\left(u_{1}, u_{2}, v\right) \in \mathbb{R}^{3}: u_{1}>0\right\}
\end{aligned}
$$

which is a convex set. This shows that $F(x ; \cdot)$ is generalized $[-(C(x) \times$ $D(x))]$-subconvexlike on $X$, but it is not $[-(C(x) \times D(x))]$-convexlike on $X$ (see e.g., [9, 24? ]) so that Theorem 4.1 of [9] cannot be applied. The Lagrangian function associated with the given problem is

$$
\begin{aligned}
\mathcal{L}_{x}(y, \theta, \lambda) & =-\theta_{1} f_{1}(x, y)-\theta_{2} f_{2}(x, y)-\lambda g(x, y) \\
& =-\theta_{1} y_{1}-\theta_{2} y_{2}-\lambda y_{1}^{3}, \quad \forall\left(\theta_{1}, \theta_{2}, \lambda\right) \in\left(-\mathbb{R}_{+}\right) \times \mathbb{R}_{+} \times \mathbb{R}_{+} .
\end{aligned}
$$

Choosing $\left(\theta_{1}^{*}, \theta_{2}^{*}, \lambda^{*}\right)=(-1,0,0)$ yields

$$
\mathcal{L}_{x}\left(y, \theta^{*}, \lambda^{*}\right)=y_{1} \geq 0=\mathcal{L}_{x}\left(x, \theta^{*}, \lambda^{*}\right), \quad \forall\left(y_{1}, y_{2}\right) \in X .
$$

Moreover, $0=\mathcal{L}_{x}(x, \theta, \lambda)=\mathcal{L}_{x}\left(x, \theta^{*}, \lambda^{*}\right), \quad \forall(\theta, \lambda) \in(C(x))^{*} \times(D(x))^{*}$, i.e. $\left(x, \theta^{*}, \lambda^{*}\right)$ is a saddle point for $\mathcal{L}_{x}$ on $X \times(C(x))^{*} \times(D(x))^{*}$, according to Theorem 3.2.

Furthermore, note that (8) is fulfilled, indeed qri $[\operatorname{conv}(g(x, X)-D(x)]=\mathbb{R}$, which guarantees the existence of $\left(\theta_{1}^{*}, \theta_{2}^{*}, \lambda^{*}\right) \in\left((C(x))^{*} \backslash\{\mathbf{0}\}\right) \times(D(x))^{*}$ in the saddle point condition according to Theorem 3.3. By Theorem 3.4, it follows that $x=(0,0)$ is a solution to qr-weak VQEP.

We next give a sufficient optimality condition for VQEP under the assumption that $-f(x, \cdot)$ is a strict $C(x)$-function in correspondence of a solution $x$ to VQEP.

Theorem 3.5 Suppose that qri $D(x) \neq \emptyset, \operatorname{cl}(C(x)-C(x))=Y, \operatorname{cl}(D(x)-$ $D(x))=Z$, (8) holds and $-f(x, \cdot)$ is a strict $C(x)$-function on the set $K(x)$ which is supposed to be convex. If there exist $\theta^{*} \in(C(x))^{*}$ and $\lambda^{*} \in(D(x))^{*}$ such that $\left(x, \theta^{*}, \lambda^{*}\right)$ is a saddle point for $\mathcal{L}_{x}$ on $X \times(C(x))^{*} \times(D(x))^{*}$, then $x$ is a solution to VQEP. 
Proof By the proof of Theorem 3.4 (i), there exist $\theta^{*} \in(C(x))^{*} \backslash\{\mathbf{0}\}$ and $\lambda^{*} \in(D(x))^{*}$ such that (7) holds. Therefore,

$$
-\left\langle\theta^{*}, f(x, y)\right\rangle \geq\left\langle\lambda^{*}, g(x, y)\right\rangle \geq 0, \quad \forall y \in K(x) .
$$

Since $-f(x, \cdot)$ is a strict $C(x)$-function on $K(x)$ and $\theta^{*} \neq \mathbf{0}$, from Lemma 2.1 it follows that the function $\psi_{x}(y):=-\left\langle\theta^{*}, f(x, y)\right\rangle$ is strictly convex on $K(x)$. Indeed, by Definition 2.3 and Lemma 2.1 it follows that, for every $x_{1}, x_{2}$ with $x_{1} \neq x_{2}$ and $\left.\alpha \in\right] 0,1[$,

$$
\begin{aligned}
& \alpha \psi_{x}\left(x_{1}\right)+(1-\alpha) \psi_{x}\left(x_{2}\right)-\psi_{x}\left(\alpha x_{1}+(1-\alpha) x_{2}\right) \\
= & \alpha\left\langle\theta^{*}, f\left(x_{1}\right)\right\rangle+(1-\alpha)\left\langle\theta^{*}, f\left(x_{2}\right)\right\rangle-\left\langle\theta^{*}, f\left(x, \alpha x_{1}+(1-\alpha) x_{2}\right)\right\rangle \\
= & \left\langle\theta^{*}, c\right\rangle>0
\end{aligned}
$$

where the last inequality follows from the fact that $c \in$ qri $C(x)$ and $\theta^{*} \in$ $(C(x))^{*} \backslash\{\boldsymbol{0}\}$. Since $-\left\langle\theta^{*}, f(x, x)\right\rangle=0$, from (15) one has $x$ is a global minimum point for $\psi_{x}$ on $K(x)$ and since $\psi_{x}$ is strictly convex on $K(x)$, it follows that $x$ is the unique minimum point on $K(x)$, which yields

$$
\psi_{x}(y)=-\left\langle\theta^{*}, f(x, y)\right\rangle>0, \quad \forall y \in K(x) \backslash\{x\} .
$$

Suppose to the contrary that $x$ is not a solution to VQEP. Then there exists $\tilde{y} \in K(x) \backslash\{x\}$ such that $f(x, \tilde{y}) \in C_{0}(x)$, so that

$$
\psi_{x}(\tilde{y})=-\left\langle\theta^{*}, f(x, \tilde{y})\right\rangle \leq 0,
$$

which contradicts (16) and therefore the proof is complete.

Example 3.2 Let $X:=\mathbb{R}, Y:=\mathbb{R}^{2}, C(x):=\mathbb{R}_{+}^{2}, D(x):=-\mathbb{R}_{+}$. Define $f(x, y):=\left(f_{1}(x, y), f_{2}(x, y)\right):=\left(-x y(y-x),-x(y-x)^{2}\right), \quad g(x, y):=x y-x$.

Let $x:=1 \in K(x)=\{y \in \mathbb{R}: y \leq 1\}$. Note that $-f(x, \cdot)$ is a strict $C(x)$ function on $K(x)$ since each $-f_{i}(x, \cdot)$ is strictly convex on $K(x), i=1,2$. Moreover, it is easy to see that (8) is fulfilled, since qri[conv $(g(x, X)-$ $D(x)]=\mathbb{R}$. The Lagrangian function associated with the given problem is

$$
\mathcal{L}_{x}(y, \theta, \lambda)=\theta_{1}\left(y^{2}-y\right)+\theta_{2}(y-1)^{2}-\lambda(y-1),
$$

$\forall\left(\theta_{1}, \theta_{2}, \lambda\right) \in(C(x))^{*} \times(D(x))^{*}=\mathbb{R}_{+} \times \mathbb{R}_{+} \times\left(-\mathbb{R}_{+}\right)$.

Choosing $\left(\theta_{1}^{*}, \theta_{2}^{*}, \lambda^{*}\right)=(0,1,0)$ yields

$$
\mathcal{L}_{x}\left(y, \theta^{*}, \lambda^{*}\right)=(y-1)^{2} \geq 0 \geq \mathcal{L}_{x}\left(x, \theta^{*}, \lambda^{*}\right)=0, \quad \forall y \in X .
$$

Moreover, $0=\mathcal{L}_{x}(x, \theta, \lambda)=\mathcal{L}_{x}\left(x, \theta^{*}, \lambda^{*}\right), \quad \forall(\theta, \lambda) \in(C(x))^{*} \times(D(x))^{*}$, i.e. $\left(x, \theta^{*}, \lambda^{*}\right)$ is a saddle point for $\mathcal{L}_{x}$ on $X \times(C(x))^{*} \times(D(x))^{*}$. By Theorem 3.5, it follows that $x=1$ is a solution to VQEP. 
Next, we analyse VQEP under convexity and Fréchet differentiability assumptions: in such a case one has the following characterization of the saddle point condition for the Lagrangian function. Denote by $\nabla_{y}$ the Fréchet differential of $f(x, \cdot)$ and $g(x, \cdot)$, by $\nabla_{y} f(x, x)^{*}$ and $\nabla_{y} g(x, x)^{*}$ the adjoint operators of $\nabla_{y} f(x, x)$ and $\nabla_{y} g(x, x)$, respectively.

Proposition 3.1 Let $U, Y, Z$ be Banach spaces and let $X$ be an open convex set in $U$. Assume that $-f(x, \cdot)$ is Fréchet differentiable at $y=x$ and is a $C(x)$-function on $X,-g(x, \cdot)$ is Fréchet differentiable at $y=x$ and is a $D(x)$-function on $X$. Then there exist $\theta^{*} \in(C(x))^{*}$ and $\lambda^{*} \in(D(x))^{*}$, $\left(\theta^{*}, \lambda^{*}\right) \neq \mathbf{0}$, such that $\left(x, \theta^{*}, \lambda^{*}\right)$ is a saddle point for $\mathcal{L}_{x}$ on $X \times(C(x))^{*} \times$ $(D(x))^{*}$ iff it is a solution of the following system

$$
\left\{\begin{array}{l}
\nabla_{y} f(x, x)^{*} \theta+\nabla_{y} g(x, x)^{*} \lambda=\mathbf{0}, \\
\langle\lambda, g(x, x)\rangle=0, \\
g(x, x) \in D(x), \theta \in(C(x))^{*}, \lambda \in(D(x))^{*}, x \in X .
\end{array}\right.
$$

Proof (Only if) Assume that there exist $\theta^{*} \in(C(x))^{*}$ and $\lambda^{*} \in(D(x))^{*}$, $\left(\theta^{*}, \lambda^{*}\right) \neq \mathbf{0}$, such that $\left(x, \theta^{*}, \lambda^{*}\right)$ is a saddle point for $\mathcal{L}_{x}$ on $X \times(C(x))^{*} \times$ $(D(x))^{*}$. Then, with a similar proof of Theorem 3.4 (i), we have that $x \in$ $K(x)$ and that $\left\langle\lambda^{*}, g(x, x)\right\rangle=0$. By the second inequality in (13) it follows that

$$
0 \leq-\left[\left\langle\theta^{*}, f(x, y)\right\rangle+\left\langle\lambda^{*}, g(x, y)\right\rangle\right], \quad \forall y \in X .
$$

Since $f(x, x)=\mathbf{0},(18)$ yields that $x$ is a global minimum point of $\mathcal{L}_{x}\left(\cdot, \theta^{*}, \lambda^{*}\right)$ on $X$. Since $f(x, \cdot)$ and $g(x, \cdot)$ are Fréchet differentiable at $y=x$, so is the function $\mathcal{L}_{x}\left(\cdot, \theta^{*}, \lambda^{*}\right)$ and its Fréchet differential at $y=x$ is given by

$$
-\left[\nabla_{y} f(x, x)^{*} \theta^{*}+\nabla_{y} g(x, x)^{*} \lambda^{*}\right] .
$$

Since $X$ is an open convex set it follows that

$$
\mathbf{0}=\nabla_{y} \mathcal{L}_{x}\left(x, \theta^{*} ; \lambda^{*}\right)=-\left[\nabla_{y} f(x, x)^{*} \theta^{*}+\nabla_{y} g(x, x)^{*} \lambda^{*}\right] .
$$

(If) Let $\theta^{*} \in(C(x))^{*}$ and $\lambda^{*} \in(D(x))^{*},\left(\theta^{*}, \lambda^{*}\right) \neq \mathbf{0}$, such that $\left(x, \theta^{*}, \lambda^{*}\right)$ be a solution of the system (17). Since $-f(x, \cdot)$ and $-g(x, \cdot)$ are a $C(x)$ function and a $D(x)$-function on $X$, respectively, the function $y \mapsto \mathcal{L}_{x}\left(y, \theta^{*}, \lambda^{*}\right)=$ $-\left[\left\langle\theta^{*}, f(x, y)\right\rangle+\left\langle\lambda^{*}, g(x, y)\right\rangle\right]$ is convex on $X$ (see, Proposition 2.1). Now the equality $\nabla_{y} f(x, x)^{*} \theta^{*}+\nabla_{y} g(x, x)^{*} \lambda^{*}=\mathbf{0}$ implies that $\nabla_{y} \mathcal{L}_{x}\left(x, \theta^{*}, \lambda^{*}\right)=\mathbf{0}$ and thus

$$
\mathcal{L}_{x}\left(x, \theta^{*}, \lambda^{*}\right) \leq \mathcal{L}_{x}\left(y, \theta^{*}, \lambda^{*}\right), \quad \forall y \in X
$$


Since $f(x, x)=\mathbf{0}$, the complementarity condition $\left\langle\lambda^{*}, g(x, x)\right\rangle=0$ leads to

$$
\begin{aligned}
\mathcal{L}_{x}(x, \theta, \lambda) & =-[\langle\theta, f(x, x)\rangle+\langle\lambda, g(x, x)\rangle] \\
& \leq 0 \\
& =-\left[\left\langle\theta^{*}, f(x, x)\right\rangle+\left\langle\lambda^{*}, g(x, x)\right\rangle\right] \\
& =\mathcal{L}_{x}\left(x, \theta^{*} ; \lambda^{*}\right), \quad \forall(\theta, \lambda) \in(C(x))^{*} \times(D(x))^{*} .
\end{aligned}
$$

This shows that $\left(x, \theta^{*}, \lambda^{*}\right)$ is a saddle point for $\mathcal{L}_{x}$ on $X \times(C(x))^{*} \times(D(x))^{*}$.

Corollary 3.1 Let $U:=\mathbb{R}^{l}, Y:=\mathbb{R}^{n}, Z:=\mathbb{R}^{m}$ and let $X$ be an open convex set in $U$. Assume that $-f(x, \cdot):=\left(-f_{1}(x, \cdot), \cdots,-f_{n}(x, \cdot)\right)$ is differentiable at $y=x$ and is a $C(x)$-function on $X,-g(x, \cdot):=\left(-g_{1}(x, \cdot), \cdots,-g_{m}(x, \cdot)\right)$ is differentiable at $y=x$ and is a $D(x)$-function on $X$. Then, there exist $\theta^{*} \in(C(x))^{*}$ and $\lambda^{*} \in(D(x))^{*},\left(\theta^{*}, \lambda^{*}\right) \neq \mathbf{0}$, such that $\left(x, \theta^{*}, \lambda^{*}\right)$ is a saddle point for $\mathcal{L}_{x}$ on $X \times(C(x))^{*} \times(D(x))^{*}$ iff it is a solution of the following system

$$
\left\{\begin{array}{l}
\sum_{i=1}^{n} \theta_{i} \nabla_{y} f_{i}(x, x)+\sum_{j=1}^{m} \lambda_{j} \nabla_{y} g_{j}(x, x)=\mathbf{0}, \\
\langle\lambda, g(x, x)\rangle=0, \\
g(x, x) \in D(x), \theta \in(C(x))^{*}, \lambda \in(D(x))^{*}, x \in X,
\end{array}\right.
$$

From Corollary 3.1, we can prove the following result:

Theorem 3.6 Let $U:=\mathbb{R}^{l}, Y:=\mathbb{R}^{n}, Z:=\mathbb{R}^{m}$ and let $X$ be an open convex set in $U$. Let $x \in X$ and assume that $-f(x, \cdot):=\left(-f_{1}(x, \cdot), \cdots,-f_{n}(x, \cdot)\right)$ is differentiable at $y=x$ and is a $C(x)$-function on $X,-g(x, \cdot):=\left(-g_{1}(x, \cdot), \cdots,-g_{m}(x, \cdot)\right)$ is differentiable at $y=x$ and is a $D(x)$-function on $X$. Suppose that $\operatorname{int} C(x) \neq \emptyset$, int $D(x) \neq \emptyset$, and that

$$
\mathbf{0} \in \operatorname{int}[(g(x, X)-D(x))] .
$$

Then, $x$ is a solution to qr-weak VQEP (or equivalently, q-weak VQEP or weak VQEP) iff there exist $\theta^{*} \in(C(x))^{*}$ and $\lambda^{*} \in(D(x))^{*},\left(\theta^{*}, \lambda^{*}\right) \neq \mathbf{0}$, such that $\left(x, \theta^{*}, \lambda^{*}\right)$ is a solution of the system (19).

Proof We observe that, in finite dimensional spaces, qi $C(x)=\operatorname{int} C(x)$, qi $D(x)=$ int $D(x)$ and int $C(x) \neq \emptyset$, int $D(x) \neq \emptyset$ imply that qri $C(x)=\operatorname{int} C(x)$, qri $D(x)=$ $\operatorname{int} D(x)$ and moreover, $\operatorname{cl}(C(x)-C(x))=\mathbb{R}^{n}, \operatorname{cl}(D(x)-D(x))=\mathbb{R}^{m}$.

(Only if) Assume that $x$ is a solution to qr-weak VQEP. The assumptions guarantee that the system (2) is image convex. By Theorem 3.1 there exists 
$\theta^{*} \in C(x)^{*}$ such that $\left(x, \theta^{*}, \lambda^{*}\right)$ is a saddle point for $\mathcal{L}_{x}$ on $X \times C(x)^{*} \times D(x)^{*}$. By Corollary $3.1\left(x, \theta^{*}, \lambda^{*}\right)$ is a solution of system (19).

(If) Assume that $\left(x, \theta^{*}, \lambda^{*}\right)$ is a solution of system (19). By Corollary 3.1, $\left(x, \theta^{*}, \lambda^{*}\right)$ is a saddle point for $\mathcal{L}_{x}$ on $X \times(C(x))^{*} \times(D(x))^{*}$ and by Theorem 3.4 we complete the proof.

Remark 3.3 Theorem 3.6 can be extended to the case where $\operatorname{int} D(x)=\emptyset$. In such a case, we need the additional assumption $\mathcal{L}_{x}\left(\bar{x}, \theta^{*}, \lambda^{*}\right)>0$, for some $\bar{x} \in X$, in order to apply Theorem 3.4 (ii) for proving the (If) part.

\section{Gap Functions and Error Bounds}

In this section, we will deepen the analysis of gap functions for VQEP (res., qr-weak VQEP) following the line considered in [9, 11, 29, 30]. In particular, we will prove that the solution set of VQEP (res., qr-weak VQEP) admits an error bound with respect to the gap function under suitable generalized convexity and strong monotonicity assumptions.

Denote by $X_{0}:=\{x \in X: x \in K(x)\}$ the domain of VQEP (res., qrweak VQEP). We suppose that $X_{0} \neq \emptyset$. Denote by $S_{0}, S_{\mathrm{qr}}, S_{\mathrm{q}}, S_{\mathrm{r}}$ and $S_{\text {int }}$ the solution set of VQEP, qr-weak VQEP, q-weak VQEP, r-weak VQEP and weak VQEP, respectively.

Definition 4.1 A function $\psi: X_{0} \rightarrow \mathbb{R} \cup\{+\infty\}$ is said to be a gap function for VQEP (res., qr-weak VQEP) if it satisfies the following properties:

(1) $\psi(x) \geq 0, \quad \forall x \in X_{0}$;

(2) $\psi\left(x^{*}\right)=0$, if and only if $x^{*} \in S_{0}$ (res., $S_{\mathrm{qr}}$ ).

In the following, we suppose that $U, Y, Z$ are Banach spaces and we set $d(x, E):=\inf _{y \in E}\|x-y\|$, where $x \in U$ and $E \subset U$ is a nonempty subset.

Definition 4.2 Let $\psi: X_{0} \rightarrow \mathbb{R} \cup\{+\infty\}$ be a gap function for VQEP. We say that an error bound holds for $S_{0}$ with respect to $\psi$ and $X_{0}$ if there exists $\mu>0$ such that

$$
d\left(x, S_{0}\right) \leq \mu \psi(x), \quad \forall x \in X_{0} .
$$

Similarly, we can define the error bounds for the solution set of qr-weak VQEP with respect to $\psi$ and $X_{0}$. 
For $x \in X_{0}$, suppose that $(C(x))^{*} \times(D(x))^{*}$ has a weakly* compact base, denoted by $B(x)$. For $x \in X_{0}$ and $(\theta, \lambda) \in B(x)$, let

$$
\begin{aligned}
\varphi(x, \theta, \lambda) & :=\sup _{y \in X}[-\mathcal{L}(x ; y, \theta, \lambda)] \\
& =\sup _{y \in X}[\langle\theta, f(x, y)\rangle+\langle\lambda, g(x, y)\rangle] .
\end{aligned}
$$

Define $\phi: X_{0} \rightarrow \mathbb{R} \cup\{+\infty\}$ by

$$
\phi(x):=\min _{(\theta, \lambda) \in B(x)} \varphi(x, \theta, \lambda), \quad \forall x \in X_{0} .
$$

We observe that the function $(\theta, \lambda) \mapsto \varphi(x, \theta, \lambda)$ is convex and weakly* continuous, since it is the supremum of a collection of linear functions. Thus, the function $\phi$ defined above, is the optimal value of a parametric problem on a weakly* compact set, with a convex objective function.

Definition 4.3 Let $P$ be a convex cone in $Y$ and $e \in \operatorname{int} P \neq \emptyset$. A mapping $h: X \times X \rightarrow Y$ is said to be $P$-e-strongly monotone on $X$ with modulus $\delta>0$ if for any $x, y \in X$,

$$
h(x, y)+h(y, x) \leq_{P}-\delta\|x-y\|^{2} e .
$$

If we let $Y:=\mathbb{R}^{n}, P:=\mathbb{R}_{+}^{n}, e_{n}:=(1, \cdots, 1)$ and let $h:=\left(h_{1}, \cdots, h_{n}\right):$ $X \times X \rightarrow Y$, where $h_{i}: X \times X \rightarrow \mathbb{R}$ for each $i=1, \cdots, n$, then $h$ is $\mathbb{R}_{+}^{n}-e_{n}$-strongly monotone on $X$ with modulus $\delta>0$ if and only if for each $i=1, \cdots, n, h_{i}$ is strongly monotone on $X$ with modulus $\delta>0$. If let $Y:=\mathbb{R}, P:=\mathbb{R}_{+}$and $e_{1}:=1$, then the above definition collapses to the classical definition of strong monotonicity of $h$ on $X$.

Theorem 4.1 Assume that $U, Y, Z$ are Banach spaces, and assume that for each $x \in S_{\mathrm{qr}}$, the mapping $F(x ; \cdot)$ defined in $(4)$ is generalized $(C(x) \times D(x))$ subconvexlike on $X$ and the generalized Slater condition (8) holds. Additionally, suppose that for each $x \in S_{\mathrm{qr}}$, any of the following two assumptions is fulfilled:

(a) $\operatorname{int} C(x) \neq \emptyset$ and $\operatorname{int} D(x) \neq \emptyset$;

(b) $\operatorname{cl}(C(x)-C(x))=Y, \operatorname{cl}(D(x)-D(x))=Z$ and $\mathbf{0} \notin$ qi $[\operatorname{cl}(\operatorname{cone}(F(x, X)-$ $(C(x) \times D(x))))]$.

Then, the following assertions hold: 
(i) The function $\phi$ is a gap function for qr-weak VQEP;

(ii) Furthermore, assume that $S_{\mathrm{qr}}:=\left\{x^{*}\right\}$ and for any $x, y \in X_{0}, g(x, y)+$ $g(y, x) \geq_{D\left(x^{*}\right)} \mathbf{0}$ and suppose that $-f$ is $C\left(x^{*}\right)$-e-strongly monotone on $X_{0}$ with modulus $\delta>0$, where $e \in$ qri $C\left(x^{*}\right)$. Then there exists $\left(\theta^{*}, \lambda^{*}\right) \in B\left(x^{*}\right)$, with $\theta^{*} \neq \mathbf{0}$, such that an error bound holds for $S_{\mathrm{qr}}$ with respect to the gap function $r(x):=\left(\varphi\left(x, \theta^{*}, \lambda^{*}\right)\right)^{\frac{1}{2}}$ and $X_{0}$.

Proof (i) We preliminarily note that for each $x \in S_{\mathrm{qr}}$, assumption (a) implies that $\operatorname{cl}(C(x)-C(x))=Y, \operatorname{cl}(D(x)-D(x))=Z$.

Let $x \in X_{0}$. Then $x \in K(x)$, i.e., $g(x, x) \geq_{D(x)} \mathbf{0}$. Since $f(x, x)=\mathbf{0}$, we have

$$
\langle\theta, f(x, x)\rangle+\langle\lambda, g(x, x)\rangle=\langle\lambda, g(x, x)\rangle \geq 0, \quad \forall(\theta, \lambda) \in B(x),
$$

and therefore $\phi(x) \geq 0, \forall x \in X_{0}$.

Let $x \in S_{\mathrm{qr}}$. Then, it follows from Theorem 3.2 that there exists $\left(\theta^{*}, \lambda^{*}\right) \in(C(x))^{*} \times(D(x))^{*}$, with $\left(\theta^{*}, \lambda^{*}\right) \neq \mathbf{0}$, such that the point $\left(x, \theta^{*}, \lambda^{*}\right)$ is a saddle point for $\mathcal{L}_{x}$ on $X \times(C(x))^{*} \times(D(x))^{*}$. Since $B(x)$ is a base of $(C(x))^{*} \times(D(x))^{*}$ and taking into account that $\mathcal{L}_{x}\left(x, \theta^{*}, \lambda^{*}\right)=0$ (see the proof of Theorem 3.4 (i)), it follows that,

$$
\begin{aligned}
\phi(x) & =\min _{(\theta, \lambda) \in B(x)} \varphi(x, \theta, \lambda) \\
& =\min _{(\theta, \lambda) \in B(x)} \sup _{y \in X}[\langle\theta, f(x, y)\rangle+\langle\lambda, g(x, y)\rangle] \\
& =\min _{(\theta, \lambda) \in B(x)} \sup _{y \in X}\left[-\mathcal{L}_{x}(y, \theta, \lambda)\right] \\
& =-\mathcal{L}_{x}\left(x, \theta^{*}, \lambda^{*}\right)=0 .
\end{aligned}
$$

Let $\phi(x)=0$ with $x \in K(x)$. Then there exists $\left(\theta^{*}, \lambda^{*}\right) \in B(x)$ such that (7) holds. From the proof of Theorem 3.1 one has $\left(x, \theta^{*}, \lambda^{*}\right)$ is a saddle point for $\mathcal{L}_{x}$ on $X \times(C(x))^{*} \times(D(x))^{*}$. Applying Theorem 3.4 (i) it follows that $x \in S_{\mathrm{qr}}$.

(ii) Let $x^{*} \in S_{\mathrm{qr}}:=\left\{x^{*}\right\}$. Then, from (i) there exists $\left(\theta^{*}, \lambda^{*}\right) \in B\left(x^{*}\right)$ such that:

$$
\left\langle\theta^{*}, f\left(x^{*}, x\right)\right\rangle+\left\langle\lambda^{*}, g\left(x^{*}, x\right)\right\rangle \leq 0, \quad \forall x \in X .
$$

Since $-f$ is $C\left(x^{*}\right)$-e-strongly monotone on $X_{0}$ with modulus $\delta>0$,

$$
f\left(x, x^{*}\right)+f\left(x^{*}, x\right) \geq_{C\left(x^{*}\right)} \delta\left\|x-x^{*}\right\|^{2} e, \quad \forall x \in X_{0} .
$$


Since $g\left(x, x^{*}\right)+g\left(x^{*}, x\right) \geq_{D\left(x^{*}\right)} \mathbf{0}, \forall x \in X_{0}$, from (20) and (21) it follows that

$$
\begin{aligned}
\varphi\left(x, \theta^{*}, \lambda^{*}\right) & =\sup _{y \in X}\left[\left\langle\theta^{*}, f(x, y)\right\rangle+\left\langle\lambda^{*}, g(x, y)\right\rangle\right] \\
& \geq\left\langle\theta^{*}, f\left(x, x^{*}\right)\right\rangle+\left\langle\lambda^{*}, g\left(x, x^{*}\right)\right\rangle \\
& \geq-\left\langle\theta^{*}, f\left(x^{*}, x\right)\right\rangle+\delta\left\langle\theta^{*}, e\right\rangle\left\|x-x^{*}\right\|^{2}+\left\langle\lambda^{*}, g\left(x, x^{*}\right)\right\rangle \\
& \geq \delta\left\langle\theta^{*}, e\right\rangle\left\|x-x^{*}\right\|^{2}+\left\langle\lambda^{*}, g\left(x, x^{*}\right)+g\left(x^{*}, x\right)\right\rangle \\
& \geq \delta\left\langle\theta^{*}, e\right\rangle\left\|x-x^{*}\right\|^{2}, \forall x \in X_{0} .
\end{aligned}
$$

By the generalized Slater condition (8), Proposition 5.4 in [14] implies that $\theta^{*} \neq \mathbf{0}$ in (20). Since $e \in$ qri $C\left(x^{*}\right)$, it follows from (i) of Lemma 2.1 that $\left\langle\theta^{*}, e\right\rangle>0$ and so $\delta\left\langle\theta^{*}, e\right\rangle>0$. Consequently, inequality (22) yields

$$
d\left(x, S_{\mathrm{qr}}\right)=\left\|x-x^{*}\right\| \leq \frac{\left(\varphi\left(x, \theta^{*}, \lambda^{*}\right)\right)^{\frac{1}{2}}}{\left(\delta\left\langle\theta^{*}, e\right\rangle\right)^{\frac{1}{2}}}=\frac{1}{\left(\delta\left\langle\theta^{*}, e\right\rangle\right)^{\frac{1}{2}}} r(x), \quad \forall x \in X_{0} .
$$

Next, we prove $r(x)=0$, if and only if $x \in S_{\mathrm{qr}}:=\left\{x^{*}\right\}$. Let $x=x^{*}$. From (20) it follows that

$$
\varphi\left(x^{*}, \theta^{*}, \lambda^{*}\right)=\sup _{y \in X}\left[\left\langle\theta^{*}, f\left(x^{*}, y\right)\right\rangle+\left\langle\lambda^{*}, g\left(x^{*}, y\right)\right\rangle\right]=0
$$

and so $r\left(x^{*}\right)=0$. If $r(x)=0$, then from (23) one has $x \in S_{\mathrm{qr}}:=\left\{x^{*}\right\}$. Theorem 4.1 improves and generalizes Theorem 5.1 in [9].

We next analyse some particular cases where it is possible to extend Theorem 4.1 to VQEP. We need the following preliminary result.

Proposition 4.1 Assume that any of the following assumptions hold:

(a) $\operatorname{int} C(x) \neq \emptyset$ and $-f(x, \cdot)$ is a strict $C(x)$-function on $K(x)$ which is supposed to be convex, for any $x \in S_{0}$;

(b) $S_{\mathrm{qr}}=\left\{x^{*}\right\}$ and $S_{0} \neq \emptyset$.

Then, $S_{\mathrm{qr}}=S_{0}$.

Proof We only need to prove that $S_{\mathrm{qr}} \subseteq S_{0}$. Assume that (a) holds and let $x \in S_{\mathrm{qr}}$, i.e., $x \in K(x)$ and

$$
f(x, y) \notin \operatorname{qri} C(x), \quad \forall y \in K(x) .
$$


Since $-f(x, \cdot)$ is a strict $C(x)$-function on $K(x)$, one has $-f(x, K(x))+$ qri $C(x)$ is a convex set. From $(24)$ and the assumption int $C(x) \neq \emptyset$, it follows that

$\mathbf{0} \notin-f(x, K(x))+\operatorname{qri} C(x)=-f(x, K(x))+\operatorname{int}(\operatorname{int} C(x))=\operatorname{int}(-f(x, K(x))+\operatorname{int} C(x))$.

Note that $\operatorname{cl}(\operatorname{int} C(x))=\operatorname{cl} C(x)=C(x)$. Then by the separation theorem for convex sets (see, for example, [31]), there exists $\theta^{*} \in Y^{*} \backslash\{\mathbf{0}\}$ such that

$$
\left\langle\theta^{*},-f(x, y)+c\right\rangle \geq 0, \quad \forall y \in K(x), \forall c \in \operatorname{int} C(x)
$$

and as a consequence,

$$
\left\langle\theta^{*},-f(x, y)+c\right\rangle \geq 0, \quad \forall y \in K(x), \forall c \in C(x) .
$$

Setting $y=x$ in (25) yields $\theta^{*} \in(C(x))^{*}$, while setting $c:=\mathbf{0}$ yields

$$
-\left\langle\theta^{*}, f(x, y)\right\rangle \geq 0, \quad \forall y \in K(x) .
$$

Arguing as in the proof of Theorem 3.5, we obtain that $x \in S_{0}$.

Now assume that (b) holds and let $x \in S_{0}$. Since $S_{0} \subseteq S_{\mathrm{qr}}$ and $x \in$ $S_{\mathrm{qr}}=\left\{x^{*}\right\}, x=x^{*}$ and therefore $S_{\mathrm{qr}}=S_{0}$.

By the previous proposition, we immediately obtain the following result.

Corollary 4.1 Assume that $U, Y, Z$ are Banach spaces, and assume that for each $x \in S_{0}$, the mapping $F(x ; \cdot)$ defined in $(4)$ is generalized $(C(x) \times$ $D(x)$ )-subconvexlike on $X$ and the generalized Slater condition (8) holds. Additionally, suppose that for each $x \in S_{0}$, the following two assumptions are fulfilled:

(a) $\operatorname{int} C(x) \neq \emptyset$ and $\operatorname{int} D(x) \neq \emptyset$;

(b) $-f(x, \cdot)$ is a strict $C(x)$-function on $K(x)$ which is supposed to be convex.

Then, the function $\phi$ is a gap function for VQEP.

Proof It follows from Theorem 4.1 taking into account that, by Proposition 4.1 we have that $S_{\mathrm{qr}}=S_{0}$.

Similarly, we can obtain an error bound for VQEP.

Theorem 4.2 Assume that $U, Y, Z$ are Banach spaces, $S_{0} \neq \emptyset, S_{\mathrm{qr}}=\left\{x^{*}\right\}$ and assume that the mapping $F\left(x^{*} ; \cdot\right)$ defined in (4) is generalized $\left(C\left(x^{*}\right) \times\right.$ $D\left(x^{*}\right)$ )-subconvexlike on $X$ and the generalized Slater condition (8) holds. Additionally, suppose that any of the following two assumptions is fulfilled: 
(a) $\operatorname{int} C\left(x^{*}\right) \neq \emptyset$ and $\operatorname{int} D\left(x^{*}\right) \neq \emptyset$;

(b) $\operatorname{cl}\left(C\left(x^{*}\right)-C\left(x^{*}\right)\right)=Y, \operatorname{cl}\left(D\left(x^{*}\right)-D\left(x^{*}\right)\right)=Z$ and $\mathbf{0} \notin$ qi $\left[\operatorname{cl}\left(\operatorname{cone}\left(F\left(x^{*}, X\right)-\left(C\left(x^{*}\right) \times D\left(x^{*}\right)\right)\right)\right)\right]$.

Then the following assertions hold:

(i) The function $\phi$ is a gap function for VQEP;

(ii) Furthermore, assume that for any $x, y \in X_{0}, g(x, y)+g(y, x) \geq_{D\left(x^{*}\right)} \mathbf{0}$ and suppose that $-f$ is $C\left(x^{*}\right)$-e-strongly monotone on $X_{0}$ with modulus $\delta>0$, where $e \in \operatorname{qri} C\left(x^{*}\right)$. Then, there exists $\left(\theta^{*}, \lambda^{*}\right) \in B\left(x^{*}\right)$, with $\theta^{*} \neq \mathbf{0}$, such that an error bound holds for $S_{0}$ with respect to the gap function $r(x):=\left(\varphi\left(x, \theta^{*}, \lambda^{*}\right)\right)^{\frac{1}{2}}$ and $X_{0}$.

Proof It follows from Theorem 4.1 taking into account that, by Proposition 4.1 we have that $S_{\mathrm{qr}}=S_{0}$.

The following example is given to illustrate Theorems 4.1 and 4.2 .

Example 4.1 Let $U:=\mathbb{R}^{l}, Y:=\mathbb{R}^{n}, Z:=\mathbb{R}^{m}, X:=[-1,1]^{l}, C(x):=$ $\mathbb{R}_{+}^{n}, D(x):=\mathbb{R}_{+}^{m}$ and let $f(x, y):=\langle x, x-y\rangle e_{n}$ and $g(x, y):=2 x-y$ for any $x, y \in X$, where $e_{n}:=(1, \cdots, 1) \in \operatorname{int} C(x)$. It is easy to check $X_{0}:=[0,1]^{l}$ and $S_{\mathrm{qr}}=S_{0}=\{\mathbf{0}\}$, the assumption (a) and other assumptions of Theorems 4.1 and 4.2 hold. Let $B(x)=\left\{z \in(C(x))^{*} \times(D(x))^{*}:\left\langle e_{n+m}, z\right\rangle=1\right\}$ for every $x \in X_{0}$, where $e_{n+m}:=(1, \cdots, 1) \in \operatorname{int}\left((C(x))^{*} \times(D(x))^{*}\right)^{*}=$ int $(C(x) \times D(x))$. Note that $B(x)$ is a base for $(C(x))^{*} \times(D(x))^{*}$ for every $x \in X_{0}$ (see, e.g., [1, Lemma 1.28]). As a consequence, the function $\phi$ is a gap function for qr-weak VQEP and VQEP and there exists $\left(\theta^{*}, \lambda^{*}\right) \in B(\mathbf{0})$ such that an error bound holds for $S_{\mathrm{qr}}$ and $S_{0}$ with respect to the gap function $r(x):=\left(\varphi\left(x, \theta^{*}, \lambda^{*}\right)\right)^{\frac{1}{2}}$ and $X_{0}$, where $\theta^{*}:=\left(\frac{1}{n}, \cdots, \frac{1}{n}\right)$ and $\lambda^{*}:=\mathbf{0}$.

\section{Conclusions}

We have investigated five kinds of vector quasi-equilibrium problems, say VQEP, qr-weak VQEP, q-weak VQEP, r-weak VQEP and weak VQEP. Specially, we have provided Lagrangian type optimality conditions and gap functions for VQEP and qr-weak VQEP under mild generalized convexity assumptions. We have also given some error bounds for the solution set of VQEP and qr-weak VQEP under mild generalized convexity and strong monotonicity assumptions. 
A further research direction to be developed in a future work might be the analysis of saddle point conditions for VQEP having infinite-dimensional image and with the ordering cones or the cones appearing in the constraints possibly having empty quasi-interior.

Moreover, it would be of interest to analyze gap functions and error bounds in the above mentioned cases and under weaker assumptions than the strong monotonicity of the operator.

Acknowledgements The research was supported by the National Natural Science Foundation of China, Project 11371015; the Innovation Team of Department of Education of Sichuan Province, Project 16TD0019; and the Meritocracy Research Funds of China West Normal University.

\section{References}

[1] Jahn, J.: Vector Optimization. Theory, Applications, and Extensions. Springer-Verlag, Berlin, Heidelberg (2011)

[2] Luc, D.T.: Theory of Vector Optimization. Springer-Verlag, Berlin (1989)

[3] Giannessi, F. (ed.): Vector Variational Inequalities and Vector Equilibria. Kluwer Academic Publishers, Dordrecht, Boston, London (2000)

[4] Blum, E., Oettli, W.: From optimization and variational inequalities to equilibrium problems. Math. Stud. 63, 1-23 (1993)

[5] Ansari, Q.H., Chan, W.K., Yang, X.Q.: The system of vector quasi-equilibrium problems with applications. J. Global Optim. 29, 45-57 (2004)

[6] Ansari, Q.H., Yao, J.C.: On Vector Quasi-Equilibrium Problems in Equilibrium Problems and Variational Models, Edited by P. Daniele, F. Giannessi and A. Maugeri, Kluwer Academic Publishers, Dordrecht-Boston-London, 118 (2003)

[7] Chen, G.Y., Huang, X.X., Yang, X.Q.: Vector Optimization. Set-valued and Variational Analysis. Springer-Verlag, Berlin (2005)

[8] Mastroeni, G.: On the image space analysis for vector quasi-equilibrium problems with a variable ordering relation. J. Global Optim. 53, 203-214 (2012)

[9] Guu, S.M., Li, J.: Vector quasi-equilibrium problems: separation, saddle points and error bounds for the solution set. J. Global Optim. 58, 751-767 (2014)

[10] Li, S.J., Teo, K.L., Yang, X.Q., Wu, S.Y.: Gap functions and existence of solutions to generalized vector quasi-equilibrium problems. J. Global Optim. 34, 427-440 (2006) 
[11] Mastroeni, G., Panicucci, B., Passacantando, M., Yao, J.C.: A separation approach to vector quasi-equilibrium problems: saddle point and gap function. Taiwanese J. Math. 13, 657-673 (2009)

[12] Mastroeni, G.: Gap functions for equilibrium problems, J. Glob. Optim. 27, 411-426 (2003)

[13] Giannessi, F.: Constrained Optimization and Image Space Analysis. Springer, New York (2005)

[14] Li, J., Mastroeni, G.: Image convexity of generalized systems and applications. J. Optim. Theory Appl. 169, 91-115 (2016)

[15] Borwein, J.M., Lewis, A.S.: Partially Finite Convex Programming, part I: Quasi-relative Interiors and Duality Theory. Math. Program. Ser. A 57, 15-48 (1992)

[16] Limber, M.A., Goodrich, R.K.: Quasi interiors, Lagrange multipliers, and $L^{p}$ spectral estimation with lattice bounds. J. Optim. Theory Appl. 78, 143-161 (1993)

[17] Bot, R.I., Csetnek, E.R., Wanka, G.: Regularity conditions via quasirelative interior in convex programming. SIAM J. Optim. 19, 217-233 (2008)

[18] Bao, T.Q., Mordukhovich B.S.: Relative Pareto minimizers for multiobjective problems: existence and optimality conditions. Math. Program., Ser. A 122, 301-347 (2010)

[19] Flores-Bazán, F., Mastroeni, G.: Strong Duality in Cone Constrained Nonconvex Optimization. SIAM J. Optim. 23, 153-169 (2013)

[20] Borwein, J.M., Goebel, R.: Notions of relative interior in Banach spaces. J. Math. Sci. 115, 2542-2553 (2003)

[21] Hiriart-Urruty, J.B., Lemaréchal, C.: Convex Analysis and Minimization Algorithms. Vol. I, Springer-Verlag, Berlin, Germany (1993)

[22] Zhou, Z.A., Yang, X.M.: Optimality conditions of generalized subconvexlike set-valued optimization problems. J. Optim. Theory Appl. 150, 327-340 (2011)

[23] Giannessi, F.: Theorems of the alternative and optimality conditions. J. Optim. Theory Appl. 60, 331-365 (1984)

[24] Mastroeni, G., Rapcsák, T.: On Convex Generalized Systems. J. Optim. Theory Appl. 104, 605-627 (2000)

[25] Bot, R.I., Csetnek, E.R., Moldovan A.: Revisiting some duality theorems via the quasirelative interior in convex optimization, J. Optim. Theory Appl. 139, 67-84 (2008) 
[26] Bot, R.I., Csetnek, E.R.: Regularity conditions via generalized interiority notions in convex optimization: new achievements and their relation to some classical statements, Optimization 61, 35-65 (2012)

[27] Giannessi, F., Mastroeni, G.: Separation of sets and Wolfe duality. J. Global Optim. 42, 401-412 (2008)

[28] Holmes, R.B.: Geometric Functional Analysis and Its Applications. SpringerVerlag, New York-Heidelberg (1975)

[29] Li, J., Huang, N.J.: Image space analysis for vector variational inequalities with matrix inequality constraints and applications. J. Optim. Theory Appl. 145, 459-477 (2010)

[30] Li, J., Huang, N.J.: Image space analysis for variational inequalities with cone constraints and applications to traffic equilibria. Sci. China Math. 55, 851-868 (2012)

[31] Zălinescu, C.: Convex Analysis in General Vector Spaces. World Scientific Publishing Co., Inc., River Edge, NJ (2002) 